\title{
NÍVEL DE ATIVIDADE FÍSICA E FATORES ASSOCIADOS EM DOCENTES DA FACULDADE DE MEDICINA DE UMA UNIVERSIDADE DO SUDOESTE GOIANO
}

\section{PHYSICAL ACTIVITY LEVEL AND ASSOCIATED FACTORS IN PROFESSORS OF THE FACULTY OF MEDICINE OF A SOUTHWESTERN UNIVERSITY IN GOIÁS}

\author{
Beatriz Nascimento Vieira ${ }^{1}$ \\ Sâmara Huang Bastos ${ }^{2}$ \\ Amanda Ferreira França ${ }^{3}$ \\ João Carvalho do Nascimento ${ }^{4}$ \\ Carlabianca Cabral de Jesus Canevari ${ }^{5}$ \\ Renato Canevari Dutra da Silva ${ }^{6}$
}

\begin{abstract}
Resumo: $O$ ritmo de trabalho e o estilo de vida moderna influenciam em baixos níveis de atividade física, o que pode ser observado nos professores universitários. Essa situação pode comprometer a saúde desses indivíduos, aumentando o risco para inúmeras doenças. Desse modo, objetivou-se com esse estudo avaliar o nível de atividade física e fatores associados em docentes da Faculdade de Medicina da Universidade de Rio Verde. A pesquisa foi realizada através dos seguintes instrumentos: Questionário Internacional de Atividade Física (versão curta), questionário para coleta do índice de massa corpórea autorreferido e de informações sociodemográficas, profissionais e de saúde e aferição da circunferência abdominal com fita métrica. Com isso, os resultados foram positivos para a correlação entre sexo e nível de atividade física, sendo que mulheres apresentam menores níveis do que os homens. Já as outras variáveis não mostraram significância estatística nessa amostra. Também, foram detectados que mais de $50 \%$ dos docentes possuem níveis insuficientes de atividade física, medidas elevadas de circunferência abdominal e índice de massa corpórea concernente com excesso de peso. Portanto, conclui-se que grande parte dos docentes apresentou um nível de atividade física insuficiente e dentre os fatores analisados, o sexo foi o único que demonstrou correlação significativa com a inatividade física.
\end{abstract}

Palavras-chave: Atividade Física. Obesidade. Circunferência Abdominal. Índice de Massa Corpórea.

Abstract: Work rhythm and modern lifestyle lead to low levels of physical activity, which can be observed in university professors. This situation can compromise the health of these individuals, increasing the risk for numerous diseases. Thus, this study aimed to evaluate the level of physical activity and associated factors in

\footnotetext{
1 Graduanda do curso de Medicina pela Universidade de Rio Verde (UniRV), E-mail: bia.nv@hotmail.com.

2 Graduanda do curso de Medicina pela Universidade de Rio Verde (UniRV), E-mail: samarahuangb@gmail.com.

3 Graduanda do curso de Medicina pela Universidade de Rio Verde (UniRV), E-mail: amandafranca10@hotmail.com.

4 Mestre em Ciências da Saúde pela UnB - Universidade de Brasília (UnB), E-mail: joao.carvalho.nascimento@hotmail.com.

5 Pós-graduação em Direito Processual Civil pela Universidade Anhanguera - Uniderp, E-mail: carlabiancacanevari@gmail.com.

${ }^{6}$ Doutorando em Saúde Coletiva, Universidade do Vale do Rio dos Sinos (UNISINOS), E-mail: renatocanevari@yahoo.com.br.
} 
professors of the Faculty of Medicine of the University of Rio Verde. The research was conducted employing the following instruments: International Physical Activity Questionnaire (short version), questionnaire for self-reported body mass index and sociodemographic, health and professional information, and measurement of waist circumference with a tape measure. Thus, the results were positive for the correlation between gender and physical activity level, with women presenting lower levels than men. The other variables did not show statistical significance in this sample. Also, it was detected that more than $50 \%$ of the professors have insufficient levels of physical activity, high measures of waist circumference and body mass index showing overweight. Therefore, it is concluded that most of the professors had an insufficient level of physical activity and among the factors analyzed, gender was the only one that showed a significant correlation with physical inactivity.

Key words: Physical Activity. Obesity. Waist Circumference. Body Mass Index.

Data de submissão: 10.11 .2019

Data de aprovação: 28.01.2021

Identificação e disponibilidade:

(https://revista.univap.br/index.php/revistaunivap/article/view/2420, http://dx.doi.org/10.18066/revistaunivap.v27i53.2420).

\section{INTRODUÇÃO}

A inatividade física é algo proeminente no atual contexto da saúde e é observada mundialmente em cerca de $17 \%$ dos adultos. Ela é responsável por elevar o risco de morbidade e mortalidade da população e repercute diretamente na saúde das pessoas, relacionando-se com várias disfunções orgânicas, como doenças cardiovasculares, diabetes, dislipidemias, câncer e obesidade (ORGANIZAÇÃO PAN-AMERICANA..., 2003).

No contexto brasileiro, observa-se que a idade cronológica do indivíduo e os níveis de atividades físicas são inversamente proporcionais e o sedentarismo é mais prevalente em adultos e idosos, principalmente em posições socioeconômicas mais baixas (FREIRE et al., 2014). Segundo estudo do IBGE, realizado em 2015, 62,1\% da população brasileira analisada não eram praticantes de esportes ou atividades físicas. Observou-se também que existia uma relação positiva entre maior grau de instrução e maior poder aquisitivo com um elevado percentual de indivíduos ativos (INSTITUTO..., 2017).

A atividade física oferece benefícios físicos, sociais, mentais e cognitivos para as pessoas, independente do gênero e idade. É uma grande aliada no que concerne aos fatores protetores cardiovasculares e metabólicos, além de prevenir hábitos viciosos como tabagismo. Os padrões de atividade física também contribuem diretamente para regulação fisiológica de peso corporal, sendo que seus baixos níveis resultam em ganho de peso e desenvolvimento de obesidade (ORGANIZAÇÃO PAN-AMERICANA..., 2003; WORLD..., 2000).

Nesse sentido, o excesso de peso, que inclui sobrepeso e obesidade, mostrase como um grande problema de saúde pública que apresenta evolução crescente ao longo das décadas. Esse aumento é decorrente de múltiplos fatores biopsicossociais que interagem entre si, resultando em uma condição complexa, que 
é simultaneamente compreendida como doença e fator de risco para outras doenças. Dessa maneira, a obesidade é considerada um agente predisponente para outros distúrbios crônicos metabólicos, como hipertensão arterial e diabetes mellitus (MALTA et al, 2014; DIAS et al., 2017).

Dessa maneira, é relevante que a distribuição de gordura corporal é um fator preditivo de saúde, e a combinação de massa corporal e distribuição de gordura são de grande importância na avaliação clínica, visto que a gordura visceral é o principal fator de risco para alterações metabólicas e doenças cardiovasculares. $O$ índice de massa corporal (IMC) é o indicador mais usado para medida da adiposidade corporal, é simples, prático e de baixo custo. Este índice é calculado através da divisão do peso $(\mathrm{kg})$ pela altura (metros) elevada ao quadrado $\left(\mathrm{kg} / \mathrm{m}^{2}\right)$. A mensuração da circunferência abdominal (CA) apresenta-se como melhor índice antropométrico para avaliação de gordura intra-abdominal, se associando muito com a gordura corporal total. A CA define-se por ser a medida no ponto médio entre a crista ilíaca e o rebordo costal inferior e é de aferição simples e reprodutível (WORLD..., 2000; ASSOCIAÇÃO..., 2016).

$O$ início da vida profissional, a falta de tempo e o estilo de vida moderno influenciam diretamente na parada da prática de exercícios físicos, o que leva a uma redução de gasto energético favorecendo o aparecimento de sobrepeso e obesidade (ASSOCIAÇÃO..., 2016). Isso pode ser levado em consideração nos professores universitários, visto que estes possuem um estilo de vida considerado sedentário, pois, muitas vezes, praticam atividades físicas de baixa intensidade (OLIVEIRA et al., 2011).

Portanto, por meio desse projeto de pesquisa objetivou-se, avaliar o nível de atividade física e os fatores associados em docentes da Faculdade de Medicina da Universidade de Rio Verde-Goiás.

\section{MATERIAIS E MÉTODOS}

Trata-se de um estudo epidemiológico transversal, de base universitária realizado na Universidade de Rio Verde - UniRV. A população analisada foi constituída pelos docentes da Faculdade de Medicina da UniRV.

O estudo respeita os critérios éticos estabelecidos pela Resolução nº 466/2012 (BRASIL, 2013) do Conselho Nacional de Saúde e recebeu aprovação do Comitê de Ética em Pesquisa da Universidade de Rio Verde, com o número de parecer 2.646.377, sendo iniciada a coleta de dados posteriormente à aprovação.

Todos os docentes foram informados, através do Termo de Consentimento Livre e Esclarecido (TCLE), sobre a natureza do estudo, objetivos, possíveis riscos, benefícios, procedimentos executados e outras informações relevantes da pesquisa. Dessa maneira, foi solicitada a assinatura do TCLE a todos que concordaram em participar da pesquisa. Foram incluídos no estudo os professores que exerceram suas atividades no ano de 2018, de ambos os sexos, sem limites de faixa etária. Dentre os critérios de exclusão foram considerados: professores apenas do internato, docentes que foram afastados durante o ano de 2018 e gestantes. Assim como, professores que possuíam doenças agudas ou crônicas que tivessem impacto significativo na coleta de dados e portadores de deficiências físicas que impedem a prática de atividades físicas, interferindo eventualmente nos resultados da coleta do nível de atividade física (NAF).

Para análise do NAF foi utilizado o Questionário Internacional de Atividade 
Física (IPAQ-versão 8) na forma curta. O questionário é composto por oito perguntas abertas acerca do tempo que cada pessoa gasta praticando atividades físicas moderadas e vigorosas em uma semanal habitual e o tempo despendido de inatividade física (MATSUDO et al., 2001).

Os questionários foram entregues aos participantes ao longo dos meses de novembro e dezembro de 2018. As perguntas foram respondidas individualmente sem intervenções dos pesquisadores, já que o questionário é autoexplicativo. Antecedendo o IPAQ, havia perguntas acerca de informações sociodemográficas, profissionais e de saúde, para coleta das variáveis independentes.

A medida da circunferência abdominal foi obtida com o uso de fita métrica nos indivíduos em posição ortostática, no ponto médio entre o rebordo costal inferior e a crista ilíaca. Os pontos de corte considerados foram os sugeridos pela Organização Mundial de Saúde (OMS) para o risco aumentado de complicações metabólicas: igual ou superior a $94 \mathrm{~cm}$ em homens e $80 \mathrm{~cm}$ em mulheres (WORLD..., 2000).

O IMC autorreferido foi calculado a partir das informações de peso e altura disponibilizadas pelos participantes nos questionários. Como ponto de corte para 0 IMC foi adotada a classificação segundo a OMS: eutrófico $(18,5-24,99)$, sobrepeso $(25-29,99)$ e obesidade $(\geq 30)$ (WORLD, 2000).

Após a coleta dos dados, todas as informações foram organizadas em planilhas e encaminhadas para o tratamento estatístico. A entrada dos dados foi realizada usando-se o programa Epi Info (Centers for Disease Control and Prevention, Atlanta, Estados Unidos), em dupla entrada e posterior comparação, para se eliminar a probabilidade de erros de digitação. A análise univariada dos dados foi realizada utilizando-se o programa SPSS, versão 21.0 (SPSS Inc., Chicago, Estados Unidos). O efeito de delineamento e a correção das medidas de efeito, com os respectivos intervalos de confiança e testes de associação foram calculados no programa Stata, versão 7.0 (Stata Corp., College Station, Estados Unidos).

A relação do nível de atividade física com as variáveis independentes foi realizada através do Teste qui-quadrado de Pearson, e as médias da circunferência abdominal por meio do Teste $t$ de Student.

\section{RESULTADOS}

No período de realização da pesquisa, o corpo docente da Faculdade de Medicina de Rio Verde possuía 116 professores, destes, 54 (46,55\%) concordaram em participar do estudo. Além disso, 32,76\% do total de professores fizeram parte dos critérios de exclusão e $5,17 \%$ se recusaram a participar da pesquisa. Devido à grande variação de cronograma entre as disciplinas, não foi possível encontrar todos os docentes na instituição durante o período de coleta dos dados, e por esse motivo, $15,52 \%$ dos indivíduos não foram apurados na pesquisa. Dessa forma, o total de indivíduos avaliados foi de 54 professores (46,55\% da amostra total).

Observou-se que os participantes possuíam de 28 a 63 anos de idade, sendo a maior parcela $(57,4 \%)$ na faixa etária de $31-40$ anos. A maioria dos docentes $(74,1 \%)$ afirmou possuir graduação em Medicina. Além disso, 31 docentes $(57,4 \%)$ eram do sexo masculino e $23(42,6 \%)$ do sexo feminino. Também foram observados que $98,1 \%$ dos docentes não eram tabagistas (tabela 1 ).

Em relação à carga horária, mais da metade dos professores $(51,9 \%)$ possuem uma carga horária de 40 horas semanais (tabela 1). Grande parte das mulheres 
apresentou uma carga horária maior $(69,57 \%)$ ou igual $(8,7 \%)$ a 40 horas semanais, já entre os homens, esses valores foram menores, $38,71 \%$ e $6,45 \%$ respectivamente (tabela 2).

Em relação ao IMC, constatou-se que $42,6 \%$ dos docentes possuem sobrepeso e 16,7\% são obesos. Do mesmo modo, os índices de CA mostraram-se aumentados para $68,5 \%$ da amostra (tabela 1 ).

Tabela 1 - Características sociodemográficas e de saúde de docentes da Faculdade de Medicina da Universidade de Rio Verde - Goiás.

\begin{tabular}{|c|c|c|c|}
\hline & & $\mathbf{n}$ & $\%$ \\
\hline Sexo & $\begin{array}{l}\text { Feminino } \\
\text { Masculino }\end{array}$ & $\begin{array}{l}23 \\
31\end{array}$ & $\begin{array}{l}42,6 \\
57,4\end{array}$ \\
\hline Idade & $\begin{array}{c}20-30 \\
31-40 \\
41-50 \\
>50\end{array}$ & $\begin{array}{c}10 \\
31 \\
10 \\
3\end{array}$ & $\begin{array}{c}18,5 \\
57,4 \\
18,5 \\
5,6\end{array}$ \\
\hline Curso formação & $\begin{array}{l}\text { Medicina } \\
\text { Outros }\end{array}$ & $\begin{array}{l}40 \\
14\end{array}$ & $\begin{array}{l}74,1 \\
25,9\end{array}$ \\
\hline Carga horária & $\begin{array}{c}<20 \mathrm{~h} \\
20 \mathrm{~h} \\
30 \mathrm{~h} \\
40 \mathrm{~h} \\
>40 \mathrm{~h}\end{array}$ & $\begin{array}{c}5 \\
12 \\
5 \\
28 \\
4\end{array}$ & $\begin{array}{c}9,3 \\
22,2 \\
9,3 \\
51,9 \\
7,4\end{array}$ \\
\hline Tabagismo & $\begin{array}{l}\text { Não } \\
\text { Sim }\end{array}$ & $\begin{array}{c}53 \\
1\end{array}$ & $\begin{array}{c}98,1 \\
1,9\end{array}$ \\
\hline IMC & $\begin{array}{l}\text { Eutrófico } \\
\text { Sobrepeso } \\
\text { Obesidade }\end{array}$ & $\begin{array}{c}22 \\
23 \\
9\end{array}$ & $\begin{array}{l}40,7 \\
42,6 \\
16,7\end{array}$ \\
\hline CA & $\begin{array}{c}\text { Normal } \\
\text { Aumentada }\end{array}$ & $\begin{array}{l}17 \\
37\end{array}$ & $\begin{array}{l}31,5 \\
68,5\end{array}$ \\
\hline NAF & $\begin{array}{c}\text { Ativos } \\
\text { Não ativos }\end{array}$ & $\begin{array}{l}24 \\
30 \\
\end{array}$ & $\begin{array}{l}44.45 \% \\
55,55 \% \\
\end{array}$ \\
\hline Total & & 54 & 100 \\
\hline
\end{tabular}

Fonte: Elaborado pelos autores. 
Tabela 2 - Características sociodemográficas e de saúde de acordo com o sexo em docentes da Faculdade de Medicina da Universidade de Rio Verde - Goiás.

\begin{tabular}{|c|c|c|c|c|c|}
\hline & & Feminino & $\%$ & Masculino & $\%$ \\
\hline Idade & $\begin{array}{c}20-30 \\
31-40 \\
41-50 \\
>50\end{array}$ & $\begin{array}{c}7 \\
12 \\
2 \\
2\end{array}$ & $\begin{array}{c}30,43 \\
52,17 \\
8,70 \\
8,70\end{array}$ & $\begin{array}{c}3 \\
19 \\
8 \\
1\end{array}$ & $\begin{array}{c}9.68 \\
61,29 \\
25,80 \\
3,23\end{array}$ \\
\hline $\begin{array}{l}\text { Curso } \\
\text { formação }\end{array}$ & $\begin{array}{c}\text { Medicina } \\
\text { Outros }\end{array}$ & $\begin{array}{c}16 \\
7\end{array}$ & $\begin{array}{l}69,57 \\
30,43\end{array}$ & $\begin{array}{c}24 \\
7\end{array}$ & $\begin{array}{l}77,41 \\
22,59\end{array}$ \\
\hline $\begin{array}{l}\text { Carga } \\
\text { horária }\end{array}$ & $\begin{array}{c}<20 \mathrm{~h} \\
20 \mathrm{~h} \\
30 \mathrm{~h} \\
40 \mathrm{~h} \\
>40 \mathrm{~h}\end{array}$ & $\begin{array}{c}1 \\
3 \\
1 \\
16 \\
2\end{array}$ & $\begin{array}{c}4,35 \\
13,04 \\
4,35 \\
69,57 \\
8,70\end{array}$ & $\begin{array}{c}4 \\
9 \\
4 \\
12 \\
2\end{array}$ & $\begin{array}{c}12,90 \\
29,04 \\
12,90 \\
38,71 \\
6,45\end{array}$ \\
\hline Tabagismo & $\begin{array}{l}\text { Não } \\
\text { Sim }\end{array}$ & $\begin{array}{c}23 \\
0\end{array}$ & $\begin{array}{c}100 \\
0\end{array}$ & $\begin{array}{c}30 \\
1\end{array}$ & $\begin{array}{c}96,77 \\
3,23\end{array}$ \\
\hline IMC & $\begin{array}{l}\text { Eutrófico } \\
\text { Sobrepeso } \\
\text { Obesidade }\end{array}$ & $\begin{array}{l}16 \\
7 \\
0\end{array}$ & $\begin{array}{c}69,57 \\
30,43 \\
0\end{array}$ & $\begin{array}{c}6 \\
16 \\
9\end{array}$ & $\begin{array}{l}19,35 \\
51,61 \\
29,04\end{array}$ \\
\hline CA & $\begin{array}{c}\text { Normal } \\
\text { Aumentada }\end{array}$ & $\begin{array}{c}7 \\
16\end{array}$ & $\begin{array}{l}30,43 \\
69,57\end{array}$ & $\begin{array}{l}10 \\
21\end{array}$ & $\begin{array}{l}32,26 \\
67,74\end{array}$ \\
\hline NAF & $\begin{array}{c}\text { Ativos } \\
\text { Não ativos }\end{array}$ & $\begin{array}{c}6 \\
17\end{array}$ & $\begin{array}{l}26,09 \\
73,91\end{array}$ & $\begin{array}{l}18 \\
13\end{array}$ & $\begin{array}{l}58,06 \\
41,94\end{array}$ \\
\hline
\end{tabular}

Fonte: Elaborado pelos autores.

No que concerne ao nível de atividade física, 30 participantes (55,5\%) apresentaram níveis insuficientes de atividade física, sendo que 17 eram do sexo feminino e 13 do sexo masculino (tabelas 1 e 2). Com isso, realizou-se a correlação do NAF com as demais variáveis através do teste qui-quadrado de Pearson ( $p$ ) para análise de possíveis relações de significância (tabela 3). Através dos resultados podemos identificar que a única variável estatisticamente significativa foi o sexo. Os demais fatores (idade, curso de formação, carga horária, tabagismo, IMC e CA), não demonstraram correlações de significância com o nível de atividade física. 
Tabela 3 - Relação do nível de atividade física insuficiente com as variáveis independentes em docentes da Faculdade de Medicina da Universidade de Rio Verde - Goiás.

\begin{tabular}{|c|c|c|c|c|}
\hline & & $\mathbf{n}$ & $\%$ & $\mathbf{p}$ \\
\hline Sexo & $\begin{array}{c}\text { Feminino } \\
\text { Masculino }\end{array}$ & $\begin{array}{l}17 \\
13\end{array}$ & $\begin{array}{l}73,9 \\
41,9\end{array}$ & 0,019 \\
\hline Idade & $\begin{array}{c}20-30 \\
31-40 \\
41-50 \\
>50\end{array}$ & $\begin{array}{c}7 \\
18 \\
3 \\
2\end{array}$ & $\begin{array}{c}70 \\
58,1 \\
30 \\
66,7\end{array}$ & 0,293 \\
\hline Curso formação & $\begin{array}{l}\text { Medicina } \\
\text { Outros }\end{array}$ & $\begin{array}{c}21 \\
9\end{array}$ & $\begin{array}{l}52,5 \\
64,3\end{array}$ & 0,445 \\
\hline Carga horária & $\begin{array}{c}<20 \mathrm{~h} \\
20 \mathrm{~h} \\
30 \mathrm{~h} \\
40 \mathrm{~h} \\
>40 \mathrm{~h}\end{array}$ & $\begin{array}{c}4 \\
5 \\
2 \\
18 \\
1\end{array}$ & $\begin{array}{c}80 \\
41,7 \\
40 \\
64,3 \\
25\end{array}$ & 0,286 \\
\hline Tabagismo & $\begin{array}{l}\text { Sim } \\
\text { Não }\end{array}$ & $\begin{array}{c}1 \\
29\end{array}$ & $\begin{array}{r}100 \\
54,7\end{array}$ & 0,367 \\
\hline IMC & $\begin{array}{l}\text { Eutrófico } \\
\text { Sobrepeso } \\
\text { Obesidade }\end{array}$ & $\begin{array}{c}14 \\
11 \\
5\end{array}$ & $\begin{array}{l}63,6 \\
47,8 \\
55,6\end{array}$ & 0,566 \\
\hline CA & $\begin{array}{c}\text { Normal } \\
\text { Aumentada }\end{array}$ & $\begin{array}{c}7 \\
23\end{array}$ & $\begin{array}{l}41,2 \\
62,2\end{array}$ & 0,149 \\
\hline
\end{tabular}

Fonte: Elaborado pelos autores.

Também, foi realizada uma comparação entre a média da CA e o NAF dos docentes (tabela 4).

Tabela 4 - Comparação das médias de circunferência abdominal dos docentes ativos e não ativos da Faculdade de Medicina da Universidade de Rio Verde - Goiás.

\begin{tabular}{cccccc}
\hline & Atividade Física & $\mathbf{n}$ & Média & Desvio padrão & $\mathbf{p}$ \\
\hline $\begin{array}{c}\text { Valores da } \\
\text { CA }\end{array}$ & Ativo & 24 & 94,79 & $\pm 14,67$ & \\
& Não Ativo & 30 & 90,81 & $\pm 13,08$ & 0,298 \\
\hline
\end{tabular}

Fonte: Elaborado pelos autores.

\section{DISCUSSÃO}

Os resultados encontrados com a coleta do IMC mostraram que mais da metade dos docentes $(59,3 \%)$ apresentam excesso de peso $(\mathrm{IMC}>25)$. Dentre as justificativas para essa tendência, a mais significativa é a influência ambiental, uma vez que 0 estilo de vida moderno estimula o excesso de peso, na medida em que possibilita a diminuição do nível de atividade física e aumento da ingestão calórica. 
Esses padrões são decorrentes de mudanças socio-comportamentais, como diminuição das refeições feitas em casa, aumento da alimentação em redes fastfood, realização das refeições em curto espaço de tempo, assim como a falta de tempo e longas jornadas de trabalho que levam à interrupção da prática de esportes e reduzem o gasto energético diário (WORLD..., 2000; ASSOCIAÇÃO..., 2016).

De acordo com a OMS, a prevalência de sobrepeso e obesidade tem se elevado em taxas alarmantes, tanto em países subdesenvolvidos quanto em países desenvolvidos. Em 2016, foi observado em escala global que aproximadamente dois em cada cinco adultos (38,9\%) apresentavam excesso de peso (WORLD..., 2000; FAO; IFAD; UNICEF; WFP; WHO, 2019). Em outros estudos, os valores encontrados para excesso de peso em professores universitários foram de 37,6\% (OLIVEIRA FILHO; NETTO-OLIVEIRA; OLIVEIRA, 2012), 47,6\% (DEVECHIO et al, 2017) e $51,04 \%$ (OLIVEIRA et al, 2011). Dessa maneira, observa-se que os valores encontrados no presente estudo se mostraram maiores do que os disponíveis na literatura.

Ademais, foram observados valores elevados de circunferência abdominal em $68,5 \%$ da amostra, isso pode ser devido aos baixos níveis de atividade física encontrados (tabela 1), visto que existe uma forte associação entre adiposidade visceral e inatividade física, e evidências suportam uma correlação entre exercícios físicos regulares e redução da obesidade abdominal (PALEY, JOHNSON, 2018). Segundo a OMS, um alto nível de CA indica risco aumentado para doenças cardiovasculares, resistência à insulina, câncer de mama, dislipidemias, dentre outros distúrbios. Isso pode ser explicado pela maior suscetibilidade à estimulação hormonal e às alterações no metabolismo e no acúmulo de lipídios que o tecido adiposo intra-abdominal possui (WORLD..., 2000).

Os níveis de atividade física encontrados foram insuficientes para mais da metade da amostra (tabela 1). Essa situação pode estar relacionada com a falta de tempo e a extensa jornada de trabalho desses profissionais, visto que grande parte da amostra possui uma carga horária de aproximadamente 40 horas semanais (tabela 1). Também, a rotina estressante e a exigência de altos níveis de atenção, concentração e de comprometimento com os estudos são justificativas que podem interferir no nível de atividade física de docentes do ensino superior (DIAS et al, 2017).

Além disso, esses dados foram semelhantes ao estudo realizado por Dias et al (2017), em que $54,4 \%$ dos docentes analisados possuíam níveis inadequados de atividade física, quando avaliados através do questionário Baecke de atividade física habitual. Assim como, ao estudo realizado em uma cidade no sul do Brasil, com o questionário IPAQ, em que a prevalência de inatividade física entre os docentes foi de 56,6\% (OLIVEIRA FILHO; NETTO-OLIVEIRA; OLIVEIRA, 2012). Entretanto, os resultados do presente estudo, se mostram maiores quando comparados ao estudo de Santana e Peixoto (2017), que também utilizaram o questionário IPAQ e encontraram $30,7 \%$ de prevalência de inatividade física entre professores universitários.

Em inquérito realizado pelo Ministério da Saúde, em diferentes cidades brasileiras, com adultos que possuem ensino superior completo, as prevalências de inatividade física encontradas com o questionário IPAQ variaram entre $27,1 \%$ e $56,3 \%$ (BRASIL, 2004).

Desse modo, observa-se que não existe um padrão entre as taxas de atividade física em diferentes regiões e grupos populacionais, embora as mesmas mostrem-se preocupantes, visto que níveis insuficientes de atividades físicas estão associados a 
maior predisposição a doenças crônicas e metabólicas, além de uma maior incidência de doenças ocupacionais, o que interfere na qualidade de vida do professor (FREIRE et al., 2014; DIAS et al., 2017).

Esses baixos níveis de atividade física também podem estar relacionados com um declínio do gasto energético observado em diversas esferas do cotidiano moderno, como uso de transporte motorizado, equipamentos mecanizados, tanto domésticos, quanto do ambiente de trabalho, uso de elevadores, escadas rolantes e portas automáticas, aumento do tempo dispendido em comportamentos sedentários (assistir à televisão, navegar na internet), além de diversas outras atividades que são projetadas para poupar tempo e energia (WORLD..., 2000).

Ademais, quando correlacionado o NAF com as outras variáveis, foi observado que docentes do sexo feminino se apresentam menos ativas quando comparadas com docentes do sexo masculino (tabela 3). Isso pode ser justificado pelos maiores índices de carga horária apresentada, visto que 78,27\% das mulheres apresentaram uma carga horária maior ou igual a 40 horas semanais, ao passo que para os homens esse valor foi de $45,16 \%$ (tabela 2 ).

Também, esse fato pode estar relacionado com o acúmulo de tarefas atribuído às mulheres: docente, profissional de saúde, comprometimento com a família e filhos, o que é desfavorável e atinge diretamente a saúde dessas profissionais (DIAS et al., 2017).

Além disso, esses dados não diferem da realidade brasileira, visto que em estudo realizado pelo Instituto... (2017), o percentual de sedentários se mostrou maior entre as mulheres, sendo que ao analisar o total de praticantes de esporte ou atividade física, observou-se que $46,1 \%$ eram mulheres e 53,9\%, homens (INSTITUTO..., 2017). Da mesma maneira, estudos internacionais apontam tendências equivalentes (BRASIL, 2004).

Esses resultados podem estar relacionados com uma construção histórica do papel de gênero imposto pela sociedade que influencia na escolha de atividades físicas de baixa intensidade pelas mulheres. Um exemplo seria a maior participação das mulheres em atividades como caminhada e dos homens no futebol e corrida (FREIRE et al., 2014; INSTITUTO..., 2017; SA SILVA; SANDRE-PEREIRA; SALLESCOSTA, 2011).

Quando realizada a comparação entre as médias da CA e do NAF (tabela 4), observou-se que a média da circunferência abdominal entre os indivíduos ativos foi mais elevada do que nos indivíduos não ativos. A OMS considera medidas mínimas de circunferência abdominal diferentes entre os sexos, para indicação de risco de complicações metabólicas. Dessa maneira, quando levado em consideração o ponto de corte para mulheres $(80 \mathrm{~cm}$ ), em ambos os grupos (ativos e não ativos) as médias ultrapassaram esse valor. Já para o ponto de corte masculino $(94 \mathrm{~cm})$, os indivíduos inativos estavam dentro da normalidade. Entretanto, é importante considerar que a inatividade física singularmente já é um fator de risco para doenças crônicas não transmissíveis, o que não exclui este último grupo de ser suscetível a tais agravos (WORLD..., 2000; ORGANIZAÇÃO PAN-AMERICANA..., 2013).

\section{CONCLUSÃO}

Constatou-se que o nível de atividade física se mostrou insuficiente em grande parte dos docentes. Não foram encontrados fatores associados ao NAF estatisticamente significativos para esta amostra de docentes, com exceção do sexo. Para essa variável, o nível de atividade física foi menor no sexo feminino. 
Além disso, grande parte dos docentes apresentou medidas elevadas de circunferência abdominal e índice de massa corpórea concernente com excesso de peso.

Considerando esses resultados, pode-se afirmar que uma parcela significativa dos docentes analisados apresenta risco elevado para doenças crônicas não transmissíveis, principalmente as cardiovasculares. Portanto, são necessárias propostas de intervenção, como a prática de exercícios físicos e alimentação equilibrada para esses indivíduos, almejando atingir uma boa qualidade de vida.

\section{REFERÊNCIAS}

ASSOCIAÇÃO BRASILEIRA PARA O ESTUDO DA OBESIDADE E DA SÍNDROME METABÓLICA - ABESO. Diretrizes brasileiras de obesidade. São Paulo, 2016. Disponível em: https://abeso.org.br/wp-content/uploads/2019/12/DiretrizesDownload-Diretrizes-Brasileiras-de-Obesidade-2016.pdf. Acesso em: 23 mar. 2020

BRASIL, Ministério da Saúde. Inquérito domiciliar sobre comportamentos de risco e morbidade referida de doenças e agravos não transmissíveis: Brasil, 15 capitais e Distrito Federal, 2002-2003. Rio de Janeiro, 2004. Disponível em:

http://bvsms.saude.gov.br/bvs/publicacoes/inquerito_domiciliar_comportamentos_risc o_doencas_transmissiveis.pdf. Acesso em: 23 mar. 2020.

BRASIL, Ministério da Saúde. Resolução no466, de 12 de Dezembro de 2012. Diário Oficial da União, n 12, seção 1, p. 59, 2013. Disponível em:

http://conselho.saude.gov.br/resolucoes/2012/Reso466.pdf. Acesso em: 30 mar. 2021.

DEVECHIO, A. P. et al. Fatores que influenciam a hipertensão arterial sistêmica e qualidade de vida em professores universitários. Revista Archives of Health Investigation, Araçatuba, v. 6, n. 8, p. 352-358, 2017. Disponível em: http://archhealthinvestigation.com.br/ArcHI/article/view/2217/pdf. Acesso em 23 mar. 2020.

DIAS, J. et al. Prática de atividade física em docentes do ensino superior: foco na qualidade de vida. Escola Anna Nery, Rio de Janeiro, v. 21, n. 4, e20170110, 2017. Disponível em: http://www.scielo.br/scielo.php?script=sci_arttext\&pid=S1414$81452017000400233 \&$ Ing=en\&nrm=iso. Acesso em 23 mar. 2020.

DIAS, P. C. et al. Obesidade e políticas públicas: concepções e estratégias adotadas pelo governo brasileiro. Cad. Saúde Pública, Rio de Janeiro, v. 33, n. 7, 2017. Disponível em: http://www.scielo.br/scielo.php?script=sci_arttext\&pid=S0102311X2017000705001\&Ing=en\&nrm=iso. Acesso em: 23 mar. 2020.

FAO; IFAD; UNICEF; WFP; WHO. The State of Food Security and Nutrition in the World 2019. Safeguarding against economic slowdowns and downturns. Rome, FAO, 2019. Disponível em: http://www.fao.org/3/ca5162en/ca5162en.pdf. Acesso em: 23 mar. 2020.

FREIRE, R. S. et al. Prática regular de atividade física: estudo de base populacional 
no Norte de Minas Gerais, Brasil. Revista Brasileira de Medicina do Esporte, São Paulo, v. 20, n. 5, p. 345-349, out. 2014. Disponível em:

http://www.scielo.br/scielo.php?script=sci_arttext\&pid=S1517-86922014000500345. Acesso em: 23 mar. 2020.

INSTITUTO BRASILEIRO DE GEOGRAFIA E ESTATÍSTICA - IBGE. Práticas de esporte e atividade física: 2015 . Rio de Janeiro, 2017. Disponível em:

https://biblioteca.ibge.gov.br/visualizacao/livros/liv100364.pdf. Acesso em: 23 mar. 2020.

MALTA, D. C. et al. Evolução anual da prevalência de excesso de peso e obesidade em adultos nas capitais dos 26 estados brasileiros e no Distrito Federal entre 2006 e 2012. Rev. Bras. Epidemiol. São Paulo, v. 17, supl. 1, p. 267-276, 2014. Disponível em:

http://www.scielo.br/scielo.php?pid=S141590X2014000500267\&script=sci_arttext\&tln $\mathrm{g}=$ pt. Acesso em: 23 mar. 2020.

MATSUDO, S. et al. Questionário internacional de atividade física (ipaq): estudo de validade e reprodutibilidade no Brasil. Revista Brasileira de Atividade Física e Saúde. Florianópolis, v. 6, n. 2, p. 5-18, 2001. Disponível em: http://rbafs.org.br/RBAFS/article/view/931/1222. Acesso em: 23 mar. 2020.

OLIVEIRA, R. A. R. et al. Prevalência de sobrepeso e obesidade em professores da Universidade Federal de Viçosa. Fisioterapia em Movimento, Curitiba, v. 24, n. 4, p. 603-612, dez. 2011. Disponível em:

http://www.scielo.br/scielo.php?script=sci_arttext\&pid=S0103-51502011000400003. Acesso em: 23 mar. 2020.

OLIVEIRA FILHO, A.; NETTO-OLIVEIRA, E. R.; OLIVEIRA A. A. B. Qualidade de vida e fatores de risco de professores universitários. Rev. Educ. Fís, Maringá, v. 23, n. 1, p. 57-67, mar. 2012. Disponível em: http://www.scielo.br/scielo.php?pid=S198330832012000100006\&script=sci_abstract\&tlng=pt. Acesso em 23 mar. 2020.

ORGANIZAÇÃO PAN-AMERICANA DA SAÚDE - OPAS. Doenças crônicodegenerativas e obesidade: Estratégia mundial sobre alimentação saudável, atividade física e saúde. Brasília, 2003. Disponível em:

http://bvsms.saude.gov.br/bvs/publicacoes/doenca_cronico_degenerativas_obesidad e_estrategia_mundial_alimentacao_atividade_fisica.pdf. Acesso em: 23 mar. 2020.

PALEY, C. A.; JOHNSON, M. I. Abdominal obesity and metabolic syndrome: exercise as medicine? BMC Sports Science, Medicine and Rehabilitation, v. 10, p.1-8, maio 2018.

SANTANA, J. O.; PEIXOTO, S. V. Inatividade física e comportamentos adversos para a saúde entre professores universitários. Rev Bras Med Esporte, São Paulo, v. 23, n. 2, p. 103-108, abr. 2017. Disponível em: http://www.scielo.br/scielo.php?pid=S151786922017000200103\&script=sci_abstract\&tlng=pt. Acesso em: 23 mar. 2020

SA SILVA, S. P.; SANDRE-PEREIRA, G.; SALLES-COSTA, R. Fatores 
sociodemográficos e atividade física de lazer entre homens e mulheres de Duque de Caxias/RJ. Ciênc. Saúde Coletiva, Rio de Janeiro, v. 6, n. 11, p. 4491-4501, nov. 2011. Disponível em:

http://www.scielo.br/scielo.php?script=sci_arttext\&pid=S141381232011001200022\&ln $\mathrm{g}=\mathrm{en} \& \mathrm{nrm}=$ iso. Acesso em: 23 mar. 2020.

WORLD HEALTH ORGANIZATION - WHO. Obesity: preventing and managing the global epidemic: report of a WHO consultation. Genebra: WHO, 2000. Disponível em: https://apps.who.int/iris/handle/10665/42330. Acesso em: 23 mar. 2020. 